RECYT

Year 23 / No 36 / 2021 / 61-69

\title{
Human factors, artificial intelligence and autonomous cars: Perspectives for a complex implementation
}

\section{Factores humanos, inteligencia artificial y autos autónomos: Perspectivas para una implementación compleja}

\author{
Sandor B. Pereira1 *, Róber D. Botelho² \\ 1- Minas Gerais State University. Escola de Design - Rua Gonçalves Dias, 1400 - Belo Horizonte - MG, Brazil. \\ 2- Federal University of Juiz de Fora. Campus Universitário - Rua José Lourenço Kelmer, s/n - São Pedro, Juiz de Fora - MG, Brazil. \\ * E-mail: sandorbanyai@live.com
}

\section{Abstract}

The centuries-old near-inseparable human/automobile relationship faces a revolution thanks to artificial intelligence gradually creating new paradigms in terms of personal urban mobility. Still, would we be prepared to relinquish our vehicle control to autonomous systems? The main objective of this work is to elucidate the main elements of the complex relationship between human factors and artificial intelligence in the development and establishment of autonomous vehicles. Thus, this paper adopted a basic methodology with a qualitative approach with an exploratory objective and technical procedures, as well as technical procedures of a documentary and bibliographic nature. Notice that autonomous systems present plausible functioning in controlled environments, even so, in an environment with several variables and an almost infinite possibility of combinations, enforced the occurrence of failures and compromised the structuring of a mental model, based on human factors, applicable to artificial intelligence. That explains the little importance given to human factors in the planning of human/autonomous machine interactions.

Keywords: Human Factors; Artificial Intelligence; Autonomous Vehicle; Human-Machine Interaction.

\section{Resumen}

La relación humano / automóvil casi inseparable de siglos de antigüedad se enfrenta a una revolución gracias a la inteligencia artificial - IA gradualmente creando nuevos paradigmas en términos de movilidad urbana personal. Aun así, ¿estaríamos preparados para ceder completamente el control de nuestros vehículos a sistemas autónomos? El objetivo de este trabajo es dilucidar los elementos principales de la compleja relación entre los factores humanos y la inteligencia artificial en el desarrollo y establecimiento de vehículos autónomos. Así, adoptamos una metodología básica con carácter cualitativo, con un objetivo exploratorio, así como procedimientos técnicos de naturaleza documental y bibliográfica. Tenga en cuenta que los sistemas autónomos presentan un funcionamiento plausible en entornos controlados, sin embargo, en un entorno con una cantidad relevante de variables y una posibilidad casi infinita de combinaciones, ha forzado la ocurrencia de fallas y ha comprometido la estructuración de un modelo mental, basado en factores humanos, aplicable a la inteligencia artificial. Esto se debe a la poca importancia dada a los factores humanos en la planificación de las interacciones humano / máquina autónoma.

Palabras clave: Factores humanos; Inteligencia artificial; Vehículo autónomo; Interacción hombre-máquina.

\section{Introduction}

The technological revolution seen in recent decades has amazed people around the world and gained popularity through the smart devices. Our daily life is full of devices connected to their peers and to the internet, whose main objective is to free their users from boring, time-consuming and complex activities. Just as the phone industry appropriated this revolution, the automobile industry has been doing it through digitally automated systems in its products. An example of the application of these systems are, for instance, the "Cruise Control" and the "Active Park Assist", easily found in vehicles on the market (1). So that more vehicle functions can be delegated to digital automated systems, it is necessary that such vehicles and their actions should be coordinated by another type of intelligence that is alien to human intelligence. Another available path, thanks to the current technological revolu- 
tion, is the advanced stage of computational intelligence. This intelligence has proven to be successful in learning, generically speaking, and laws of physics which prevail over an environment and, therefore, analyze data captured by sensors in order to assess their development (2). This capacity has great value to the automotive industry that seeks products adapted to a scenario of intelligent and autonomous mobility of direct human action.

There are high expectations regarding the implementation of integrated vehicles in this intelligent mobility system, the two main ones related to the drastic reduction of accidents caused by human failure and the use of fuel, whether of fossil or renewable origin. Analyzing this scenario, there are estimates that by the year 2040 the highways will have lanes destined exclusively for autonomous vehicles (3). Although the optimistic outlook of these estimates could be questioned, of all the industries that have invested in automating the functions of their products, the automotive industry holds the greatest advances in this area (4). Intelligent mobility is a concept that tends to become increasingly concrete with every advance in the tests involving automotive autonomous systems guided by artificial intelligence, with inputs from the environment around them, the current traffic laws and the habits presented by human drivers in traffic.

In order to meet the growing demand for urban mobility in an increasingly chaotic road system, autonomous vehicles have been developing at a significant pace in recent years. The metropolises and megalopolises management face the transport system as a critical issue, daily transportation, industrial and commercial logistics search for the transport system even more than only from point $\mathrm{A}$ to point B mobility. Despite autonomous vehicles seen as a probable solution for traffic jams, traffic accidents and inefficient fuel consumption, ironically an interaction between the users and the vehicle ends up being excluded when designing proper technologies for autonomous vehicles (5).

Finally, it is necessary that the essential mechanisms of human factors are understood and applied to the development of autonomous digital systems for driverless vehicles. The joint planning and coordination of human and autonomous machines activities need to be synchronized during this transition period to ensure a natural and harmonious interaction of tasks (4). This important concern, as there is a transition stage between strictly human vehicle driving to strictly robotic vehicle driving that requires further study, placing the human being as a central element of this process. For this reason, it is necessary to research which elements intrinsic to human factors could guide the development of these autonomous vehicles heavily dependent on artificial intelligence in their decision-making and collaborate in understanding the complexity of human-machine interactions present in this industrial segment.

\section{Objectives and Method}

The main objective of the paper is to elucidate the major elements that interfere in the complex relationship between human factors and artificial intelligence, having as study object the $100 \%$ autonomous vehicles propositions. This objective aims to point out elements that reinforce the hypothesis that an Artificial Intelligence focused on the total control of the vehicle does not possess all the mechanisms to deal with other human drivers and the dynamics of contemporary mobility.

\section{Method}

To better gather the data that could ensure a concise analysis and a comprehensive discussion, a basic methodology, of a qualitative nature, with an exploratory objective and technical procedures of a documentary and bibliographic nature, was adopted.

As an initial step, using the virtual Google Scholar search engine, a vast bibliographic search was lead. In this step, the keywords used were: driverless cars; autonomous vehicles; artificial intelligence, and human factors. English keywords were used to access more data due to the number of publications in this language. Books, articles from periodicals, and documents already available on the internet were selected, such as magazines and news from specialized newspapers.

The next step was the selection of the sources raised in the previous step. For the Autonomous Vehicles sources, they were selected as sources whose publication date did not precede the second half of the 1990s, as it is understood that, at the time, research involving this technology was not minimally tested in environments with multiple variables. For the selection of sources that dealt with Artificial Intelligence, we opted for those that interface with ergonomics and cognitive processes. Finally, for the selection of sources related to human factors, the inclusion criterion was the focus on human-machine interaction, especially with regard to the human-vehicle relationship.

Following the guidelines for the selection of material, the following were considered as sources for the preparation of this article: 10 books; 8 book chapters; 12 articles published in journals; 3 technical standards; 1 journalistic story; 1 doctoral thesis, and 4 articles published on the internet in specialized portals. After the survey and selection of the material to be studied, critical analysis and crossing of the collected data were carried out so that, in the next step, the information could be condensed around the three areas of interest of this work: human factors; artificial intelligence and autonomous vehicles. As a next step, and also conclusive, the most relevant data were confronted with different points of view from different authors in order to corroborate the hypothesis raised in this work. 


\section{Autonomous Vehicles}

Smart mobility is a concept linked to the fourth industrial revolution. Autonomous vehicles are the most impactful components within this new mobility concept (6). What makes intelligent mobility different from all we have seen applied so far is that this new mobility is guided by complete automation, high dependence on artificial intelligence, and technology equipment on board vehicles and mirrored by streets and roads. To in-depth into the concepts covered in this work, in line with the guidelines of intelligent mobility, we decided to define an autonomous vehicle as a vehicle fully capable of receiving input from the environment around it and navigating without any human assistance (7).

There are some levels of vehicle automation functions found on the roads and in development laboratories in general. These vehicles are rated on a scale of 0 to 4 , where level 0 assumes that the driver has complete and solitary control of the primary functions - brakes, steering, acceleration, and driving force - at all times. At the last level-level 4 - the vehicle is responsible for coordinating all critical safety and operation functions, and the user is only responsible for entering the destination and authorizing departure (8).

Therefore, for this paper, the autonomous vehicles considered in the analysis and discussion were those categorized as having level 4 of automation. This option was made because those vehicles fit the description given above and for the reason which they are considered as the last stage of development of this product segment. Having as a parameter a reality transformed by new technologies, human impact on the environment, and changes in human behavior, several companies have been committed to bringing to the market a multitude of artificially intelligent assistants with the potential to simplify people's daily lives (4).

\section{Artificial Intelligence}

In general, the definition of Artificial Intelligence (AI) is born from the convergence of two complementary concepts: the first views AI as an engineering discipline dedicated to the creation of intelligent machines, the second concept defines, in turn, AI as science empirical focus on the computational development of human intelligence (2). Intelligent machines, carrying some of the intelligence-like thinking, may be able to learn new actions. This learning process would reveal a change in capabilities or behavior caused by the experience of this same machine with the environment around it (9).

Four categories expand the best definition of Artificial Intelligence and elucidate the study of this technology when applied, whether in the creation of intelligent machines or the computational development of human-like intelligence: Thinking Humanly, Thinking Rationally, Acting Humanly, and Acting Rationally (10). In the Thinking Humanly category, the AI would think like humans, which involves decision-making, problem-solving, diverse learning, etc. (11). In the Thinking Rationally category, this AI would be able to perceive, reason, and act in a way that is $100 \%$ autonomous from human action (12). In the Acting Humanly category, AI would give life to other systems whose functions depend on intelligence when performed (19). Finally, the Acting Rationally category elucidates the ability of an AI to demonstrate intelligent behavior, based on the inputs offered by the surrounding environment and the AI's prior knowledge (14).

Each year an exponential increase in data processing capacity and availability has been observed so that sophisticated algorithms can make significant progress, from accurate medical diagnosis to forecasting the population's cultural interests (15). Several forms of AI, here represented by computer programs capable of recognizing patterns and responding to multiple stimuli, are designed in our days to not depend on any human interaction to generate identical - or superior - results to those arising from a human agent (16). In parallel, there is also relevant development in the segment of intelligent personal assistants, for example, those existing in smartphones (15). The existence of these intelligent personal assistants reveals, in turn, the ability to capture, process, and combine complex and dynamic data originating from a direct human source.

\section{Human Factors}

Ergonomics as an area of knowledge covers a broader spectrum, seeking to understand the relationship between human beings and work, however, despite being treated as synonymous with Ergonomics for decades. The Human Factors area has undergone transformations that are positioning it as an independent field focused on the nature of interactions between humans and artifacts in general (17). The Human Factors area deals with the variables that impact the ability of a human being to interact with elements of a system in the search for fulfilling a settled objective or task (18).

Several factors are relevant in how human beings design strategies to accomplish a task. Some of these strategies include anthropometric characteristics analysis (related to the dimensions of the human body and its members), biomechanical characteristics (which involve the ability to produce movement and forces), and cognitive ability (related to the ability to information processing, knowledge acquiring, memory storage, and data processing for decision-making) (19). Human beings have developed, throughout their evolution, ways to extract information, either explicitly or implicitly, from tactile, sound, and visual sources of the environments and objects around them. Through these data, human beings consolidated a 
dynamic system of stimulus and feedback, essential for any activity (4).

Another human factor intrinsically present since the beginning of human civilization is symbols. The use of symbols as a vector that conveys meaning is widely used, from road signs to more abstract models used to represent complex machinery functions (20). For example, the transit system uses several symbols to communicate the norms established by social codes on which the system depends for its operation. The human mind is capable of creating, absorbing, interpreting these symbols. The human brain is also capable of making decisions based on the meaning of a symbol. Thus, the brain usually compares the stored memory based on the information with the context around these symbols (19).

\section{Discussion and analysis}

\section{About autonomous vehicles}

Initially, it is necessary to emphasize that it is not yet possible to go deeper into a more detailed analysis of autonomous vehicles, given, of course, their stage of development. Some models with Autopilot functions are far from being classified as Level 4. This difficulty of deep analysis relies on several areas besides ergonomics and engineering. Law and policy is an example where the concept of vehicles that do not depend on a driver to get around is still the subject of much debate and laws in an embryonic stage. In the United States, to mention a country where there are research and development on this type of product, few states have any legislation for autonomous vehicle use on their streets and highways (9). The delay in formulating laws and the variety of ways in which these laws impact the use of these vehicles can feed different forms of a conception of autonomous automotive systems and, therefore, imply significant forms of human-machine interaction between states in the same country.

In addition to the legislative sphere, autonomous vehicles still lack credibility among consumers of products from the automobile industry. Surveys conducted in the United States, United Kingdom, and Australia revealed that only $23.5 \%$ of people believe that autonomous vehicles (level 4) will most likely reduce the severity of car accidents (21). Users often still have strong ties to driving an automobile, and for these users, there is the fear of ceding control of the vehicle's functionalities to a computer. This fear becomes reinforced by the fact that the act of driving is potentially dangerous (22). It seems natural for users to be skeptical about the current capacity of vehicles to be fully responsible for vehicle control, given that the driver's main concerns are: reading and interpreting traffic signs; the state of a specific road; weather conditions; traffic conditions; the behavior of other drivers; pedestrian movement and existence of obstacles (23). The vehicle's intelligence systems must assume this set of variables, which demons- trates the complexity of the correct functioning of an autonomous vehicle.

Comprehensively, the actions taken by an intelligent agent, which in this case also applies to autonomous cars, will depend entirely on the perceived information (10). Thus, it is irrevocable that sensors and software present in the vehicles are capable of detecting, with exactness, your surroundings. Although an autonomous vehicle has the most accurate sensors and most advanced software, it is difficult to predict what measures it will take regarding the arbitrariness of the environment around it. The sudden appearance of a running animal or pedestrian in the vehicle's trajectory will demand reactive responses that prevent the user from understanding if the vehicle can distinguish a specific scenario from a common one (24).

The technology needed for an autonomous vehicle level 4 already exists, but the high cost of its full implementation and the infinite variables contained in traffic end up requiring different approaches and sensing technologies (25). Moreover, many roads are in poor condition. Some states like Illinois and Connecticut (USA), for instance, have $73 \%$ of the road network that needs repairs (26). The complete replacement of human factors in driving activities by autonomous automotive systems may continue to be viewed with disbelief by users and a pertinent obstacle for such systems' development.

\section{About artificial intelligence}

The AI field progression applied to autonomous vehicles becomes vital for such smart devices. This advance allows autonomous cars to have the same capacity as a human mind to connect knowledge and action. The ability of a machine to artificially reproduce these connections accurately is one of the pillars for the execution of activities, until now, exclusively human (10).

Computer science faces the In-vehicle autonomous systems as a significant challenge in this field, not only for fully controlling the execution of tasks but the interaction between these systems and their peers within complex scenarios (27). These rational agents resulting from AI advances applied to autonomous vehicles end up incorporating the area of Social Intelligence, where systems are designed to act and reason in highly unpredictable and fully open worlds. Despite the effort, these agents will start their activities with limited and uncertain knowledge; they will eventually interfere, cooperate, or actively compete with other systems (28).

Considering that an intelligent agent has the primary function of making decisions, it is necessary to emphasize that these decisions will be taken from the connections between logical reasoning - supported by a set of knowledge - and previously acquired experiences (2). Thus, the knowledge acquisition - regardless of coming from exploratory experience or preset data - will result in the cognitive processes consolidation, which is essential 
for the behavior of such agents (29). Until these autonomous vehicles can make their rational decisions given the available inputs, the time these agents will need to build their repertoire of knowledge and experiences is worrying. For the interaction of autonomous cars to be close to that performed by human beings, the vehicle must carry on it a miniaturized version of the nearby reality, where the AI can experiment with different scenarios and conclude which decision will result in the best output. This process allows the vehicle to deal with the present and the future in a more complete, safer, and more competent way in any emergency (30).

Most of the intelligent systems developed for this type of vehicle focus on keeping the car moving and perceiving the world around it. The new challenge lies in the interaction of these systems with an environment that is constantly changing, complex, thanks to other artificial networked systems, human users demanding actions, and passive human users within this same environment (31). Far beyond the intention to replace the human factors which guide the vehicle's steering, there is a significant number of systems that interact with the user, for instance: navigation systems, entertainment centers, customizable ergonomics, and comfort systems. These systems still have limited development when it comes to autonomous systems aimed at users (4). The acceptance of these intelligent agents by consumers is related to meeting several interaction demands. These same demands are born within complex traffic systems and can come from passive and active users. This fact needs attention in the development process of these systems in autonomous vehicles and, therefore, determinants in the consolidation and marketing success.

\section{About the Human Factors}

All above, the old human-automobile relationship is complex. The changing paradigm involving these automobiles may break the symbol that cars have. Regardless of gender, age or behavior, human beings have developed a unique connection between the vehicle and the driving activity (32). One of these symbols - shared by men and women - linked to the automobile is the feeling of power and freedom when enjoying a complex device that obeys your commands (33). Giving up direct control over the machine is perhaps the supplanted paradigm by the smart mobility revolution.

The arrival of autonomous technologies in the consumer market happens through luxury models. The insertion of these tools looks like a reflection of the technological race to replace human factors in vehicular driving (1), which makes it impossible to prevent such changes in urban mobility. The focus relies on the availability of capable vehicles in minimizing the driving monotony and dangerousness while reducing the side effects of urban transport (4).
About the vehicular conduction activity, between $85 \%$ and $95 \%$ of sensory signals are exclusively visual. Although most stimuli are visual, cognitive abilities such as memory, information processing, and decision-making become critical for driving a vehicle (5). The human being, during driving activities, uses his eyes, ears, and other organs to perceive the environment, as well as arms and legs to carry out control actions and a complex brain to coordinate the interrelationship of all processes. On the other hand, an autonomous vehicle has cameras, infrared and lasers to read the world around it, software to interpret situations, and motors to perform actions. As a result, the technological resources given to autonomous vehicles aim to deliver to the product a kind of omniscience of everything around it and a fast reactive capacity (10).

The insertion of sensors in places difficult to reach for human vision and the presence of super-fast processors make this omniscience a critical factor for intelligent mobility. Autonomous navigation systems developed based on image processing recognition of aesthetic patterns tend to work well under certain conditions; however, they tend to reveal alarming limitations when faced with several complex situations such as the driving act (33). An example of this is industrial robots that interact perfectly well with well-trained people in well-planned tasks. In contrast, autonomous vehicles endowed with AI will deal with relatively untrained people and with varied tasks dictated by the situations and environment where they will be inserted (4). Much progress has been made in the development and construction of autonomous systems; however, it is noteworthy that little has been reached in terms of human factors in the planning of interactions between autonomous machines and human beings. For this reason, we question the complete delivery of the functions of driving vehicles to autonomous automotive systems. A possible solution would be a kind of flexible vehicular autonomy, a new approach to an autonomous human-machine interaction in the future.

An autonomous car became a computer on wheels which its processing power would be responsible from the air conditioning system to navigation and driving. Naturally, there are certain precautions to be taken (4). The inhuman rationale will never have the same creative capacity or insight compared to the human ones; thus, it would prevent them from making any judgment (34). This abyss separates them from human beings precisely in the intentionality of decision-making, regardless of how competent such computers may be in processing data. And this lack of analysis and judgment of symbols ends up reflecting on the learning of the AI itself, and it may interfere directly in the construction of its base of experiences and, consequently, in the mental model structured by it in solving new problems. As we turn objects around us into smart devices, they end up integrating a kind of human-machine social ecosystem, which demands that 
such objects have communicative and social capabilities compatible with our interaction patterns (4). It is up to developers to balance the desire for market success for their products with the side effects that may impede the implementation of smart mobility within big cities.

\section{About the human-machine-environment}

By analyzing the chaotic cities traffic environment, there is another aspect of the human-autonomous machineenvironment interaction to be considered: complex tasks performed by autonomous vehicles demand sophisticated processing and decision-making techniques. The ability to analyze, in real-time, the status of the environment and the behavior pattern of human beings present there is the key to the safe operation of the system. For this reason, it is common for accidents caused by human error to happen. When something goes wrong, its unfolding is significantly fast, a fact that demands a short response time since many drivers have fluctuating attention levels (4).

The tendency of problems in such diverse environments is relevant when constituted by artificial and natural intelligent agents (35). Human drivers need to be able to produce comprehensive information in a natural language from a complex society. These drivers obtained their knowledge through erudition and by observing the behavior of other drivers, a fact that ends up generating a complex communicational repertoire to optimize the ability to respond to demands and establish an effective behavior (10). The same would apply to autonomous vehicles, regardless of the processing capacity or the knowledge inserted in their memory. The exploration of the environment and its consequences is part of the repertoire construction. After all, the ability of an agent to manipulate symbols governed by pre-established rules is a fundamental principle of intelligence (36). When it comes to autonomous vehicles' ability to think, it is necessary to keep in mind that it is not an easy task to work dealing with informal knowledge or with a considered number of variables. Another question rests on the simulation of situations fulfilled with hundred possible solutions as such demand can overload the available computational resources (10). These factors would prevent a vehicle from being able to respond effectively to an emergency.

Another point in the interaction dynamics between humans, autonomous cars, and the environment is the plurality of interaction elements built over decades of coexistence. Elements of natural interaction such as sounds, vibrations, lights, and smell are the basis of an implicit form of communication between vehicles and human beings (4). At the same time that autonomous vehicles attract the consumers' attention, many of these natural elements of communication seem to lose their meaning. Of course, since the user of this product will not occupy the position of control of the system. Elements that previously served to alert the driver about the state of the road or vehi- cle operation became an impediment to a new locomotion experience. Thus, the human-machine interaction context has been left behind for the technical achievement of a vehicle which drives itself (37).

Even if drivers comfortably transfer all vehicle functions control to AI, however intelligent it may seem, the automotive industry will need to invest more and more in research into how design contributes to developing a reliable relationship between users and machines (38). In addition to the great challenge of making these vehicles operational, autonomous cars will present specific challenges because transparent communication between user and machine must be a two-way street (4). The code lines and errors reported by the software will need to match in the semiotic lexicon used by drivers daily, of course, considering a scenario where autonomous vehicles will also coexist with pedestrians, cyclists, motorcyclists, and animals. Autonomous machines end up working very well inside a controlled environment or with limited possibilities for variations. When inserted in the unpredictable condition of busy streets and roads, the series of errors would increase by the precarious translation between the language of machines and the complex human communication system.

Autonomous systems that neglect such patterns of semiotic reasoning will tend to compromise the flow of interaction with the environment and the agents involved in it (35). Given this complex semiotic network unique to the human mind, to allow autonomous vehicles to think like human beings, one must first determine how they organize and categorize symbols in the moment of reflection. After mapping the human mental process, it can be possible to set it into the software. Thus, it makes an exact and randomly intentional artificial reproduction (10). Again, the dynamics of human-machine interaction end up returning to the agent's cognitive characteristics, even with sensory and mechanical features that are compatible or superior to those of humans.

For this, it is necessary to consider four groups of central interactions:

1. Human-Machine: in a lower level, since smart devices - including the autonomous vehicle itself - will become just a channel (a kind of mobile communication totem) through which users would demand their wishes, losing the current relation of total control of the machine. This relationship will be focused on the mobility service itself and user entertainment when on the move;

2. Human-Human: it will be a consequence of the previous interaction group. Of course, since the vehicle's driving function will no longer be in the care of the user. Therefore, there will be more space for the user (ex-driver) to interact with other systems and users through the car. The car will only be a mediator, as indicated in the previous item;

3. Machine-Environment: since it will be up to the autonomous vehicle and the intelligent streets/roads to 
identify, evaluate and decide which strategies to adopt when moving. The input of the variables that involve the moving vehicle, in addition to the human language code itself, should be shared and analyzed by the vehicle together with the environments adapted exclusively to support the driving activity;

4. Machine-Machine: in systems that condition displacements of subsystems (other autonomous vehicles), evaluating the intentions of other agents, inherent to the rides, and their almost infinite interrelationships with all variables in the system, will require the mobility system as a whole to behave like a large and complex multicellular organism).

Autonomous vehicles will have several interconnected systems to manage the complex performed activities. This information complexity can create an infinite range of feedbacks with too much information for the user. Some of this information may sound contradictory, demanding different answers (39). Cars that have complex systems for managing their actions may hide several routines and procedures, which leads to barriers in the complete evaluation of the system by the user. This effect can generate two problems I - lack of confidence if the vehicle system is working well, and if it is making the most coherent decisions; II - there can be blind trust in the automation if the automation fails. The user will not have the knowledge and time to avoid critical events.

Finally, the other users of the transit system who are not related to the driver-users, there is the challenge of maintaining, or optimizing, the communication of these autonomous vehicles with pedestrians, cyclists, and users of other non-autonomous vehicles. Research and development aimed at communication between autonomous systems and the surrounding human beings need consideration, especially in the current stage of these agents' creation. The entire relationship built between the elements that make up the traffic was born from the human perspective of dialogue with the available technological sources. It is enough to raise those that still make sense to human relationships and improve those that lacked improvement due to a technical issue.

\section{Conclusion}

Since the birth of the automobile, human beings have been learning to deal with these machines, assimilating their technological resources and directly interacting with their systems. With each technological advance applied to innovations within the automotive sector, new interaction references were created or reinforced in users. The speed at which sensing and computing technologies are evolving has made the concepts of autonomous mobility leave the field of science fiction to gain our reality. The capacity of these technologies is superior to the most basic human factors, such as spatial perception, understanding, and carrying out simple tasks. However, the absence of evidence that demonstrates the cognitive capacity of these technologies in autonomous vehicles raises doubts about their efficiency. One-step complete traffic automation (and its understanding) could solve many of these problems. But, it is an unlikely scenario considering the level of political, social, economic, and technological commitment.

The ability of machines to learn, combining prior knowledge and observed knowledge, associated with past experiences, still seems to be a challenge to be overcome by the AI area. The complexity of implementing autonomous vehicles with an $\mathrm{AI}$ as a substitute for human factors proved to be a significant impediment. Such machines need to deal, simultaneously, with four spheres of interaction: Human-Human, Human-Machine, Machine-Machine, and Machine-Environment. To a greater degree, the spheres of Human-Machine and Machine-Environment interaction will demand an accumulation of repertoire from computer systems.

The solution that seems to be the most suitable is what can be called a "shared control". Such "shared control" would combine the ability of Artificial Intelligence to collect and process data with human beings' skills to make decisions when adverse events occur. The situations faced by the human driver and their decision-making could contribute to the maturation of the autonomous system database, creating a repertoire of how to react to unforeseen anomalies during vehicle programming. In this scenario, the machine will be responsible for confronting the driver's choice with the environmental conditions and with the possible results of that action. After doing that, build evolutionary experiences to perform even better to avoid the repetition of situations.

Finally, even with the engineering's challenge involved in these mobile intelligent agents' development, it is impossible to deny the relevance of the human factor in the interactions of these machines within the transport chain. Autonomous vehicles would be surrounded by an environment full of dynamic elements that could respond in unpredictable ways depending on the stimulus they receive. Machine learning techniques or the simple mimicry of the human being by an AI can cause several losses along the way to reach its desired protagonism. Any mistake could stop the adoption of autonomous vehicles in the short term future.

\section{References}

1. Howley, D. I. The Race to Build Self-Driving Cars, https:// www.laptopmag.com/articles/high-tech-cars-go-mainstream-self-driving-in-car-radar-more, (2012).

2. Jordan, M. I., Russel, s. Computational intelligence. The MIT encyclopedia of the cognitive sciences, p.74-91, London (1999).

3. IEEE. You won't need a driver's license by 2040, http://si- 
tes.ieee.org/itss/2014/09/15/you-wont-need-a-driverslicense-by-2040/.

4. Norman, D. The Design of Future Things. BasicBooks, New York (2009).

5. Yang, J., Coughlin, J. F. In-vehicle technology for self-driving cars: Advantages and challenges for aging drivers. International Journal of Automotive Technology. Cambridge (2014).

6. Azmat, M. Future Scenario: Self-driving cars - the future has already begun. Viena, Unpublished (2015).

7. Gehrig, S. K., Stein, F.J. Dead reckoning and cartography using stereo vision for an autonomous car. Proceedings 1999 IEEE/RSJ International Conference on Intelligent Robots and Systems. Human and Environment Friendly Robots with High Intelligence and Emotional Quotients, pp. 1507-1512. IEEE Press, New York (1999).

8. NHTSA. U.S. Department of Transportation Releases Policy on Automated Vehicle Development, https://www. transportation.gov/briefing-room/us-department-transportation-releases-policy-automated-vehicle-development, Washington (2013).

9. Reisberg, D. Learning. The MIT encyclopedia of the cognitive science. pp. 460-461. London (1999).

10. Russel, S. J., Norving, P. Artificial intelligence: a modern approach; Pearson, New Jersey (2009).

11. Bellman, R. E. An introduction to artificial intelligence: Can computers think?. Boyd and Fraser, San Francisco (1978).

12. Winston, P. H. Artificial intelligence. Pearson, Englewood (1992).

13. Kurzwell, $\mathbf{R}$. The age of intelligent machines. MIT Press, Cambridge (1990).

14. Nilsson, N. J. Artificial intelligence: a new synthesis. Morgan Kaufmann, San Francisco (1998).

15. Schwab, K. The fourth industrial revolution, Currency, Redfen (2016).

16. Floridi, L. The 4th revollution: How the infosphere is reshaping human reality, Oxford Press, New York (2014).

17. Karwowski, w. "The discipline of human factors and ergonomics" in Handbook of Human Factors and Ergonomics, G. Salvendy, Ed., pp. 3, Hoboken (2012).

18. Chapanis, A.; garner, w.; Morgan, c. Historical foundations of human factors, in Human Factors in Simple and Complex Systems (2018), Robert W. Proctor, Trisha Van Zandt, Eds., pp. 3, Boca Raton (1949).

19. Bhise, v. D. Ergonomics in the automotive design process, CRC Press, New York (2011).

20. Helander, M. A guide to human factors and ergonomics, CRC Press, Boca Raton (2006).

21. Schoettle, B., Sivak, M. A survey of public opinion about autonomous and self-driving vehicles in the U.S., the U.K., and Australia, UMTRI-2014-21, Ann Arbor (2014).

22. Nathan, s. Autos on autopilot: the evolution of the dri- verless car, https://www.theengineer.co.uk/issues/ august-2013-online/autos-on-autopilot-the-evolutionof-the-driverless-car/\#ixzz2hJHF31Q3, (2013).

23. ETSI Technical Committee Human Factors. Human Factors (HF); Intelligent Transport Systems (ITS); ICT in cars, Report No.: ETSI TR 102762 v1.1.1., Grasse (2010).

24. Fagnant, D.J., Kockelman, K.M. Preparing a nation for autonomous vehicles: Oportunities, barriers and policy recommendation. ENO Center for Transportation, Washington (2013).

25. Ünsal, c. Intelligent navigation of autonomous vehicles in an automated highway system: Learning methods and interacting vehicles approach. Faculty of the Virginia Polytechnic Institute and State University (1997).

26. U.s. Department of Transportation: DOT fact sheets highlight grim state of U.S. roads and bridges. https:// www.transportation.gov/briefing-room/dot-fact-sheetshighlight-grim-state-us-roads-and-bridges, (2015).

27. McBurney, P., Parsons, S. Dialogue games for agent argumentation. Argumentation in Artificial Intelligence. $\mathrm{p}$. 261-280, Edinburgh (2009).

28. Castelfranchi, C. Modelling social action for AI agents. Artificial Intelligence. vol 103, issues 1-2, pp. 157-182 (1998).

29. Schneider, w. Automaticity. The MIT encyclopedia of the cognitive science. pp. 63-64. London (1999).

30. Craik, $\mathrm{K}$. The nature of explanation. Cambridge University Press, London (1943).

31. Bobrow, D. Dimensions of interaction, http://www.aaai. org/ojs/index.php/aimagazine/article/view/904/822, (1991).

32. Quinn, B. Men love cars 'because they're part of them, https://www.telegraph.co.uk/news/uknews/1557297/ Men-love-cars-because-theyre-part-of-them.html, (2007).

33. Mihaylov, M. Men love for cars: desire to control or real emotions?, https://www.montway.com/blog/men-lovefor-cars-desire-to-control-or-real-emotions, (2016).

34. Pomerleau, D. A. ALVINN: an autonomous land vehicle in a neural network. https://kilthub.figshare.com/articles/ ALVINN_an_autonomous_land_vehicle_in_a_neural_ network/6603146/1, (2018).

35. Shoham, Y., Tennenholtz, M. On social laws for artificial agent societies: offline design. Artificial Intelligence, vol. 73, No. 1-2, pp 231-252. https://www.sciencedirect.com/science/article/pii/000437029400007, (1995).

36. Winograd, T. Thinking machines: can there be? Are we? The foundations of artificial intelligence: a sourcebook. pp. 167-189, New York (1990).

37. Ju, W., Leifer, L. The design of implicit interactions: $M a-$ king interactive systems less obnoxious. Design Issues, vol. 24, No. 3, pp 72-84. MIT Press, Cambridge (2018).

38. Brownlee, J. How ui/ux design will map the future of selfdriving cars. https://www.fastcompany.com/3052738/ 
how-ui-ux-design-will-map-the-future-of-self-drivingcars, (2015).

39. Little, c. The intelligent vehicle initiative: Advancing "human-centered" smart vehicle. Federal Highway Administration Research and Technology. https:// www.fhwa.dot.gov/publications/publicroads/97septoct/ p97sept18.cfm, (1997). 